\title{
Determination of ammonium in seawater by column-switching ion chromatography
}

\author{
Yuan Huang ${ }^{\mathrm{a}}$, Shi-fen Mou ${ }^{\mathrm{a}, *}$, J.M. Riviello ${ }^{\mathrm{b}}$ \\ ${ }^{a}$ Research Center for Eco-Environmental Sciences, Chinese Academy of Sciences, P.O. Box 2871, Beijing 100085, China \\ ${ }^{\mathrm{b}}$ Dionex Corporation, Sunnyvale, CA 94086, USA
}

Received 29 June 1999; received in revised form 10 November 1999; accepted 16 November 1999

\begin{abstract}
A system which combines column switching and concentration was developed. A binary eluent mechanism was developed to study the effect of high sample concentration matrix on retention time shifts of the trace analyte. Separation conditions were chosen according to this mechanism to reduce the retention time shift of ammonium in the presence of high concentration sodium ion: high concentration sulfuric acid $(25 \mathrm{mmol} / \mathrm{l})$ was used as the eluent, and a hydronium-selective column of high capacity - a CS12 column, was employed. Since the retention time shift was reduced, the interval between onset and the complete elution of a high concentration ammonium standard $(10 \mathrm{mg} / \mathrm{l})$ was directly defined as the column-switching time window, which greatly simplified the procedure for determining the time window. Results showed that for ammonium below $1 \mathrm{mg} / 1,90 \%$ ammonium was introduced and concentrated. Detection limits of $12.8 \mu \mathrm{g} / 1$ were obtained for ammonium with sodium at $1000 \mathrm{mg} / \mathrm{l}$. (ㅇ 2000 Elsevier Science B.V. All rights reserved.
\end{abstract}

Keywords: Seawater; Column switching; Ammonium; Sodium

\section{Introduction}

Ammonia is an important micronutrient in the world's water system. High concentrations of ammonia in water can lead to nutrient enrichment of water systems, and the subsequent increase in biological activity can lead to algal blooms and the potential for turbidity, taste, odor and toxicity problems. Ammonia emitted into the atmosphere is readily trapped by acidic-cloud droplets to form the ammonium salt, or it may react with acidic gases to

*Corresponding author. Fax: +86-10-6292-3552.

E-mail address: shifenm@mail.rcees.ac.cn (S.-f. Mou) form aerosol [1]. It is also reported that ammonia plays an important role in the nitrogen cycle of shallow and eutrophicated waters [2]. Therefore, the determination of ammonia in natural water is of significant interest in air pollution control and environmental evaluation of water.

There are many methods used for the determination of ammonia or ammonium ion [3-14]. Indophenol blue spectrophotometric method is the most commonly used method [5-7]. However, this method is time consuming. Electrochemical sensors are widely used for the determination of ammonium. These methods demand specially designed devices [8-11]. By using nitrogen-14 NMR, Simeral proposed a technology, which can provide identification 
and quantitation of the important nitrogen-containing species in a single analysis [12].

Ammonium can be separated from other cations and detected by ion chromatography at the $\mu \mathrm{g} / 1$ level [15]. However, for samples containing sodium of very high concentration, such as seawater, it is difficult to quantify the concentration of trace ammonium, since ammonium and sodium ions have similar selectivities for stationary phases containing commonly used sulfonate or carboxylate cation-exchange functional groups. These cations, therefore, elute in close proximity to each other, making quantification of the ammonium difficult.

To overcome this problem, Rey et al. developed an isocratic column-switching method, which greatly increases the resolution between sodium and ammonium ions, and allows for the determination of sodium-to-ammonium concentration ratios in the order of 20000 -to- 1 . This method requires two columns containing different functional groups [16]. It is also reported that a new stationary phase containing carboxylic acid and phosphonate functional groups as well as a crown ether group, can separate very dissimilar concentration ratios of cations isocratically. In this method, some organic solvent such as acetonitrile is added to the eluent to improve the separation [17].

In recent years, 'heart-cut' column-switching techniques have been demonstrated to be powerful analytical tools [18-22]. In this method, a six-step procedure was needed to optimize the 'heart-cut' timing parameters $(\mathrm{H}-\mathrm{C}$ time window) [18].

In this paper, we report a novel column-switching technology using common cation separation columns for the determination of low concentration ammonium in seawater. Time windows can be determined directly by the standard solution of ammonium.

\section{Experimental}

\subsection{Equipment}

All system components and columns were from Dionex. (Sunnyvale, CA, USA). A Model DX-300 ion chromatograph equipped with a $25-\mu 1$ sample loop was employed along with an AI-450 chromatography workstation for instrument control as well as data acquisition and processing. An IonPac CS12A 4-mm separation column was used. Two high-pressure four-way valves and a concentrator column (CG-12A $4 \mathrm{~mm}$ ) were utilized for the column-switching technique. Detection was performed using a PED electrochemical detector in the conductivity mode. A CSRS-I cation self-regenerating suppressor was used in the external water mode. The configuration is illustrated in Fig. 1.

\subsection{Reagents}

All reagents were of analytical reagent grade unless specified otherwise. Distilled deionized water was used throughout. Solutions were filtered through a $0.45-\mu \mathrm{m}$ membrane filter and degassed before use. All ammonium standard solutions were prepared from ammonium chloride (Beijing Xinguang Chemical Reagent Factory, China). Sulfuric acid (high purity, Beijing Chemical Reagent Institute, China) was used as the eluent. The flow-rate was $1.0 \mathrm{ml} /$ $\min$.

\subsection{Preparation of real samples}

Three seawater samples were collected from China. The samples are: No. 1, from Tianjin, which is in the north of China; No. 2, from Lianyungang, Jiangsu province in the southeast of China; No. 3, from Yantai, Shandong province in the north of China. All the samples were diluted 20 times with distilled deionized water. All sample solutions were filtered through a $0.45-\mu \mathrm{m}$ filter before injection.

\subsection{Experimental procedure}

This two-stage procedure consisted of matrix elimination followed by IC analysis of ammonium. In the ammonium analysis, from 5.0 to $6.1 \mathrm{~min}$, the effluent was introduced to a concentrator column (Fig. 1A). Since the eluent (sulfuric acid) was neutralized to water in the suppressor, the effluent was collected onto the concentrator column. During the remainder of the analysis, and by switching a four-way valve (V2, Fig. 1B) the majority of the matrix and strongly retained cations in the samples were eluted from the column to waste. After all sample cations were eluted from the separation 

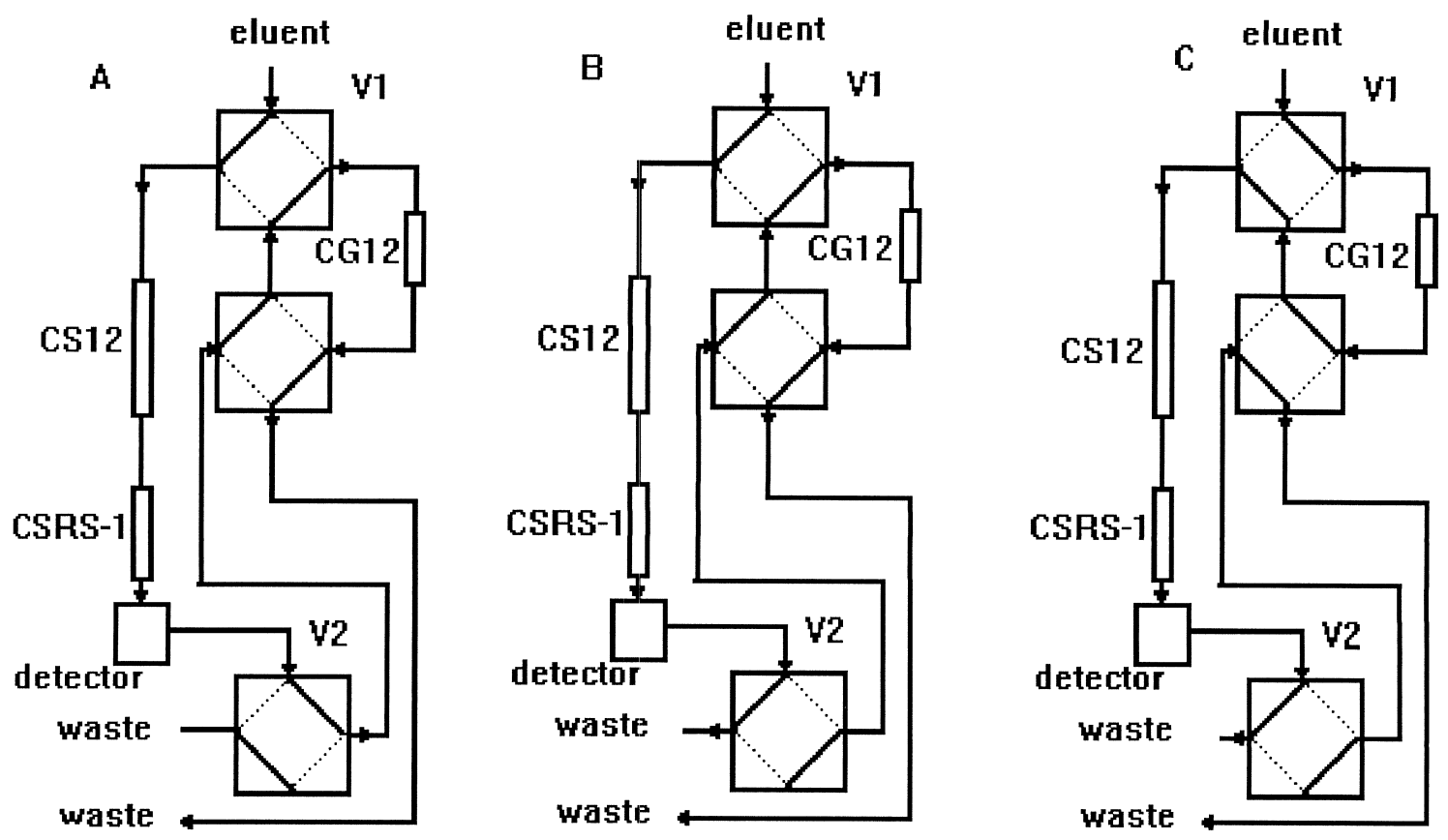

Fig. 1. System configuration. The passway is shown as the solid line.

column (15 min), the analysis of ammonium began. (Fig. 1C). The isolated ammonium was then eluted from the concentrator column. The configuration of the different valves and columns are illustrated in Fig. 1. The program for the two-stage procedure is list in Table 1.

It should be pointed out that because of the trace ammonium ion that exists in the sulfuric acid, some ammonium would be concentrated on the concentration column. To eliminate the systematic bias caused by this portion of ammonium, a blank sample (distilled deionized water) was analyzed before every

Table 1

Program for the two-stage procedure

\begin{tabular}{ccc}
\hline $\begin{array}{c}\text { Time } \\
(\mathrm{min})\end{array}$ & $V_{1}$ & $V_{2}$ \\
\hline 0.0 & On & Off \\
5.0 & On & On \\
6.1 & On & Off \\
$15.0^{\mathrm{b}}$ & Off & Off \\
25.0 & Off & Off \\
\hline
\end{tabular}

${ }^{\mathrm{a}}$ The passway is shown as the real line in Fig. 1. Eluent: 25 $\mathrm{mmol} / 1 \mathrm{H}_{2} \mathrm{SO}_{4}$, flow-rate $=1.0 \mathrm{ml} / \mathrm{min}$.

${ }^{\mathrm{b}}$ Begin data collection. real sample analysis. The data obtained is the difference between the signal of the real sample and that of the blank.

\section{Results and discussion}

\subsection{Principle}

3.1.1. The effect of sample matrix on the retention behavior of trace analyte

When a large amount of matrix ions exist, the retention time of the ammonium will shift. Novic et al. studied the effect of high concentration matrix on the retention of analyte in detail [23]. In this paper, we developed a binary eluent mechanism in which the concentration and the eluent power of both eluent and matrix ions were considered as important factors to effect the retention behavior of the analyte. The mechanism is derived as follows:

(1) The retention volume of the analyte is defined by the following equation [24]:

$V_{\mathrm{R}}=V_{\mathrm{M}}+K V_{\mathrm{S}}$ 
where $V_{\mathrm{M}}$ is the dead volume of the system, $V_{\mathrm{S}}$ is the volume of stationary phase, $K$ is the distribution coefficient of the analyte and is given by:

$K=S_{\mathrm{X}} / C_{\mathrm{X}}$

where $S_{\mathrm{X}}$ and $C_{\mathrm{X}}$ are the concentrations of analyte in stationary phase and mobile phase, respectively.

In a given chromatography system, $V_{\mathrm{M}}$ and $V_{\mathrm{S}}$ are constants. Thus, the retention volume of the analyte will directly depend on the distribution coefficient $(K)$ of the analyte.

(2) The effective column capacity (denoted as $Q$ ) is expressed by the sum of adsorbed cations, including matrix cations (denoted as A) and eluent cations (denoted as E). Since the concentration of the analyte is very small. Adsorption of the analyte is neglected [25]. According to this assumption, we have:

$Q=S_{\mathrm{E}}+S_{\mathrm{A}}$

where $S_{\mathrm{E}}$ and $S_{\mathrm{A}}$ are concentrations of the eluent cation and matrix cation in the stationary phase, respectively.

(3) Electroneutrality is maintained during the elution process. Since the concentration of counterions (anions) remains fixed at the mobile phase concentration (denoted as $U$ ), the sum of the concentration of matrix and eluent cations in the mobile phase must likewise equal $U$ so as to obey electroneutrality demands [26]. Also the concentration of analyte is neglected. According to this assumption, we have:

$C_{\mathrm{A}}+C_{\mathrm{E}}=U$

where $C_{\mathrm{A}}$ and $C_{\mathrm{E}}$ are the concentrations of the matrix ion and eluent ion in the mobile phase, respectively.

(4) For every pair of ionic species in the system, there is a constant ion-exchange equilibrium coefficient corresponding to the concentration equilibrium for the exchange reaction.

When a large amount of co-eluent ion exists in the matrix, the following equilibrium should hold for the ion-exchange resin [27]:

$S_{\mathrm{E}}=\left(K_{1} C_{\mathrm{E}}\right)\left(S_{\mathrm{X}} / C_{\mathrm{X}}\right)$

$S_{\mathrm{A}}=\left(K_{2} C_{\mathrm{A}}\right)\left(S_{\mathrm{X}} / C_{\mathrm{X}}\right)$

where $K_{1}$ and $K_{2}$ are the ion-exchange equilibrium coefficient between analyte ion and eluent ion, and between the analyte ion and the matrix ion on the ion-exchange resin. Combining Eqs. (5) and (6) with Eq. (3), one obtains:

$Q=\left(K_{1} C_{\mathrm{E}}+K_{2} C_{\mathrm{A}}\right)\left(S_{\mathrm{X}} / C_{\mathrm{X}}\right)$

Combining Eqs. (7) and (4) with Eq. (2), the distribution coefficient $(K)$ of the analyte is therefore given by:

$K=\left(S_{\mathrm{X}} / C_{\mathrm{X}}\right)=Q /\left(K_{1} U-K_{1} C_{\mathrm{A}}+K_{2} C_{\mathrm{A}}\right)$

When no matrix ion exists, the value of the distribution coefficient of the analyte, denoted as $K_{0}$, is given by:

$K_{0}=\left(S_{\mathrm{X}} / C_{\mathrm{X}}\right)=Q /\left(K_{1} U\right)$

Compare Eqs. (8) and (9), the ratio of $K_{0}$ to $K$ can be expressed as:

$$
\begin{aligned}
K_{0} / K & =\left(K_{2} C_{\mathrm{A}}-K_{1} C_{\mathrm{A}}+K_{1} U\right) /\left(K_{1} U\right) \\
& =\left[\left(K_{2} / K_{1}\right)-1\right]\left(C_{\mathrm{A}} / U\right)+1
\end{aligned}
$$

If the effect of the matrix is eliminated or effectively suppressed, the value of $K_{0} / K$ will equal or approximate 1 . Thus, we have:

$\left[\left(K_{2} / K_{1}\right)-1\right]\left(C_{\mathrm{A}} / U\right)=0$

Eq. (6) divided by Eq. (7). The value of $\left(K_{2} / K_{1}\right)$ is therefore given by:

$K_{2} / K_{1}=\left(S_{\mathrm{A}} C_{\mathrm{E}}\right) /\left(S_{\mathrm{E}} C_{\mathrm{A}}\right)$

From Eq. (12), the value of $\left(K_{2} / K_{1}\right)$ shows the preference of resin for matrix ion over the eluent ion. If this value equals to 1 , it indicates that the resin has the identical preference for these two ions.

From Eqs. (11) and (12), it shows that in order to suppress the effect of the matrix,

(1) $K_{2} / K_{1}$ approaches unity. In recent years, a special technique called on-column matrix elimination was proposed for the determination of trace analyte in the presence of high concentration matrices $[28,29]$. In this method, the matrix ion was used as a component of the eluent to overcome problems of the variable retention times and loss of chromatographic efficiency induced by a high concentration matrix. However, since the detection 
method employed must be selective, in most cases, conductimetric detection can not be used in this technique.

(2) $C_{\mathrm{A}} / U$ approaches zero. In order to suppress the peak shift, the concentration of the mobile phase $(U)$ should be as high as possible.

\subsection{Separation conditions}

Analysis time is an important factor that should be considered when choosing separation conditions. In addition, as discussed above, the eluent power and concentration of the eluent should also be considered. In this paper, columns containing carboxylate functional group were preferred, for they are hydronium-selective, in which the eluent power of hydronium is promoted [30] and the difference in eluent power between eluent and matrix ions is reduced. From Eq. (12), we know that in this case the value of $\left[\left(K_{2} / K_{1}\right)-1\right]$ can be reduced. When columns having sulfonic acid functionality are used, high concentrations of hydronium ion are required (above 40 mmol/l). Although retention time shifts may be suppressed for the sake of the high concentration eluent, it was observed that when hydronium of higher concentration was used (above $30 \mathrm{mmol} / \mathrm{l}$ ), background conductance increased to higher than 4.5 $\mu \mathrm{S}$, which means the suppressor can not provide enough capacity to convert the eluent to water. As a result, the hydronium can not be suppressed completely by the suppressor, and the residual hydronium ions will enter the concentration column and elute some ammonium from the concentration column, causing low concentration efficiency.

Two commonly used columns having carboxylate functionality, are the CS12 and CS14. When sodium is the matrix ion and hydronium is used as the eluent, these two columns have similar values of $\left(K_{1} / K_{2}\right)$, but CS12 has higher capacity than CS14. To obtain the same eluent power, the CS12 can use a higher concentration hydronium as the eluent. From Eq. (12), we can see CS12 is better than CS14.

An eluent concentration of $25 \mathrm{mmol} / 1$ sulfuric acid (flow-rate $=1.0 \mathrm{ml} / \mathrm{min}$ ) was chosen as the separation condition, in which baseline resolution of $10 \mathrm{mg} / 1$ sodium and $10 \mathrm{mg} / 1$ ammonium standard was obtained and ammonium was eluted within 6.0 min.

\subsection{The determination of column-switching time window}

To simplify the procedures for determining the time window, a relatively high concentration ammonium standard $(10 \mathrm{mg} / \mathrm{l})$ was used to determine the time window. The interval between onset and the complete elution of the standard is directly defined as the time window. There are two advantages of this method:

1. In a wide time window determined by high concentration standard solution, analyte of lower concentration can be introduced to concentration column completely.

2. The shift of the analyte peak can be included in a relatively wide time window.

It should be pointed out that when the concentration of sodium is higher than $2000 \mathrm{mg} / \mathrm{l}$, ammonium can not be separated from sodium because of the high concentration of sodium introduced to the concentration column (Fig. 2C).

\subsection{Matrix elimination}

Fig. 2A illustrates that, when attempting to detect ammonium of $50 \mu \mathrm{g} / 1$ in the presence of a high sodium level (i.e. $1000 \mathrm{mg} / \mathrm{l}$ ), the resolution is not sufficient to separate ammonium from sodium. This is demonstrated in Fig. 2B by treating the mixed standard solution $\left(50 \mu \mathrm{g} / 1 \mathrm{NH}_{4}^{+}+1000 \mathrm{mg} / 1 \mathrm{Na}^{+}\right)$. With column-switching technology, the majority of the sodium in the matrix was eliminated and the sodium level was reduced to less than $10 \mathrm{mg} / \mathrm{l}$, which is sufficient to resolve ammonium from sodium under the chosen separation conditions.

\subsection{Efficiency of the concentration column}

A series of mixed standard solutions were analyzed according to the procedure described in the experimental procedure and the recoveries of the ammonium retained on the concentration column were calculated. The mixed standard solutions consisted of $1000 \mathrm{mg} / \mathrm{l}$ sodium and ammonium of various concentrations from $50 \mu \mathrm{g} / \mathrm{l}$ to $5 \mathrm{mg} / \mathrm{l}$. Deionized water was analyzed as the blank sample before every standard solution analysis. The same column-switching time window determined accord- 

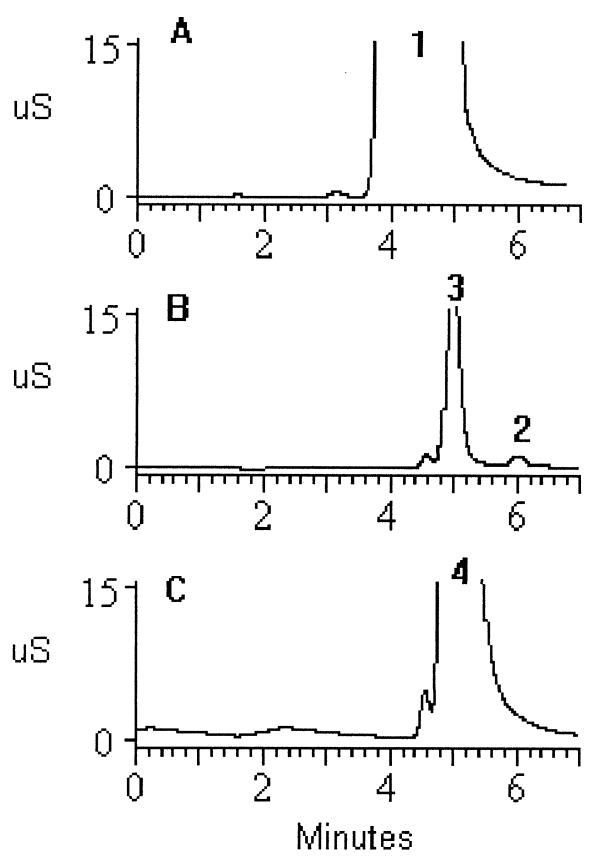

Fig. 2. Determination of ammonium in mixed standard solutions. (A) and (B) are chromatograms of mixed standard solution containing $500 \mu \mathrm{g} / 1$ ammonium and $1000 \mathrm{mg} / 1$ sodium ions. Separation conditions of (A): eluent, $25 \mathrm{mmol} / 1 \mathrm{H}_{2} \mathrm{SO}_{4}$; column: CS12; without using column switching. Separation conditions of (B): via column switching; separation conditions are list in Table 1. (C) is the chromatograms of mixed standard solution containing $500 \mu \mathrm{g} / 1$ ammonium and $2000 \mathrm{mg} / 1$ sodium ions. Separation conditions are the same with (B). Peaks: $1=\mathrm{NH}_{4}^{+}(500 \mu \mathrm{g} / 1)+$ $\mathrm{Na}^{+}(1000 \mathrm{mg} / 1) ; 2=\mathrm{NH}_{4}^{+}(500 \mu \mathrm{g} / 1) ; 3=\mathrm{Na}^{+} ; 4=\mathrm{Na}^{+}+\mathrm{NH}_{4}^{+}$ (500 $\mu \mathrm{g} / 1)$.

ing to the procedure described in Section 3.3 was used throughout.

Table 2 shows recoveries for ammonium. For 50

Table 2

Recovery (\%) of ammonium $(n=3)$

\begin{tabular}{rl}
\hline $\begin{array}{l}\text { Concentration } \\
(\mu \mathrm{g} / 1)\end{array}$ & $\begin{array}{l}\text { Recovery } \\
\text { mean } \pm \operatorname{RSD}(\%)\end{array}$ \\
\hline 50 & $97.3 \pm 6.8$ \\
100 & $98.5 \pm 4.5$ \\
200 & $99.3 \pm 2.5$ \\
500 & $99.7 \pm 0.8$ \\
1000 & $90.6 \pm 0.6$ \\
5000 & $81.2 \pm 0.4$ \\
\hline
\end{tabular}

$\mu \mathrm{g} / \mathrm{l}$ to $1 \mathrm{mg} / \mathrm{l}$ ammonium, the concentrator column retained a high percentage of the ammonium. While for $5 \mathrm{mg} / 1$ ammonium, the recoveries of the ammonium decreased greatly, indicating the concentration column overloading. The high recovery also indicated that within the time window determined directly by the standard solution, a high percentage of the ammonium was introduced to the concentration column.

\subsection{Accuracy and detection limit}

A series of standard solutions consisted of 1000 $\mathrm{mg} / \mathrm{l}$ sodium and ammonium of various concentrations from $50 \mu \mathrm{g} / 1$ to $5 \mathrm{mg} / 1$ were analyzed To eliminate the systematic bias introduced by the impurity ammonium in the sulfuric acid (reagent blank), distilled deionized water was analyzed as blank sample before each mixed sample analysis. Results of seven analyses of blank showed that the average of the blank is $223 \mu \mathrm{g} / \mathrm{l}$, and the relative standard deviation is $1.7 \%$. Each mixed standard sample was run, blank corrected in order to establish the calibration curve. Results showed that when the concentrations were from $50 \mu \mathrm{g} / 1$ to $5 \mathrm{mg} / \mathrm{l}$, correlation coefficient of the peak area was 0.9997 . The detection limits were obtained by taking seven replicate analyses of mixed standard solutions containing $50 \mu \mathrm{g} / \mathrm{l}$ ammonium and $1000 \mathrm{mg} / 1$ sodium. Then the standard deviation of the replicate analyses was calculated. The MDL was then calculated as follows [31]:

$M D L=t \times S$

where $t$ is the student's value for a $99 \%$ confidence level and a standard deviation estimate with $n-1$ degrees of freedom ( $t=3.14$ for seven replicates); $S$ is the standard deviation of the replicate analyses. The RSD based on the determination of $200 \mu \mathrm{g} / 1$ ammonium $(n=7)$ is $2.5 \%$. Detection limits was found to be $12.8 \mu \mathrm{g} / 1$.

\subsection{Analysis of samples}

Three seawaters collected from different areas of China were analyzed. Results showed that the matrices in these three seawaters did not interfere with 

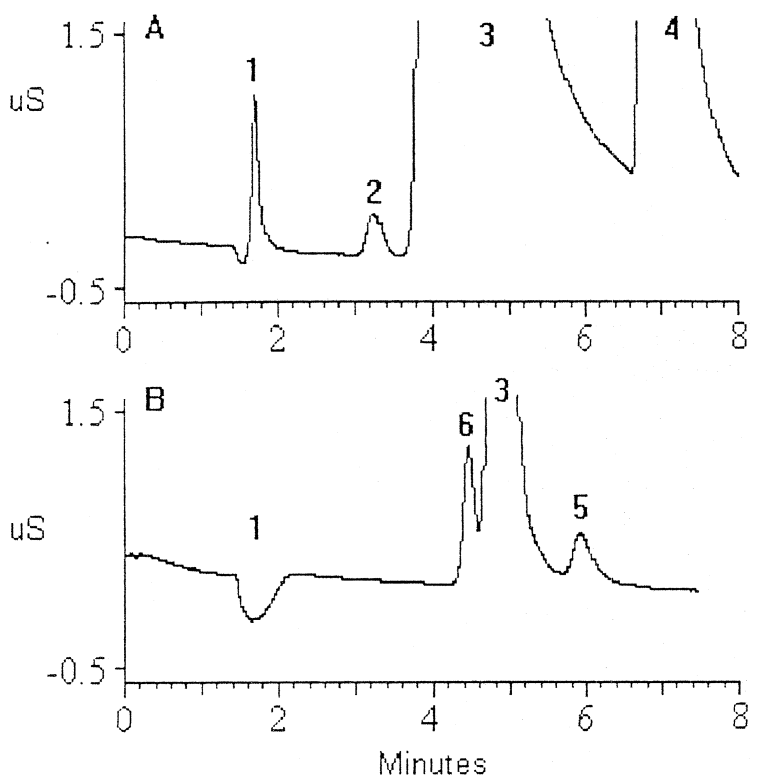

Fig. 3. Determination of ammonium in seawater. Separation conditions of (A): eluent, $25 \mathrm{mmol} / 1 \mathrm{H}_{2} \mathrm{SO}_{4}$; column: CS12; without using column switching. Separation conditions of (B): via column switching; separation conditions are list in Table 1. Peaks: $1=$ system peak; $2=\mathrm{Li}^{+} ; 3=\mathrm{Na}^{+} ; 4=\mathrm{K}^{+} ; 5=\mathrm{NH}_{4}^{+} ; 6=$ unknown.

the determination of ammonium (Fig. 3). The spike recoveries were listed in Table 3.

\section{Conclusion}

This method provides for the accurate and precise determination of ammonium at low concentrations in seawater in a relatively short time and at low cost without pretreatment. In addition, the principle of

Table 3

Determination of ammonium in diluted seawater samples $(20 \times$ dilution)

\begin{tabular}{llllcl}
\hline Sample & $\begin{array}{l}\text { Found } \\
(\mu \mathrm{g} / \mathrm{l})\end{array}$ & $\begin{array}{l}\text { Spike } \\
(\mu \mathrm{g} / \mathrm{l})\end{array}$ & $n$ & $\begin{array}{l}\text { Recovery } \\
(\%)\end{array}$ & $\begin{array}{l}\text { RSD } \\
(\%)\end{array}$ \\
\hline No. 1 & 19.2 & 150 & 5 & 94 & 3.0 \\
No. 2 & 45.0 & 250 & 6 & 107 & 2.0 \\
No. 3 & 31.3 & 200 & 5 & 104 & 2.4 \\
\hline
\end{tabular}

this method also showed that when column-switching technology is employed, the eluent concentration and the selectivity of the eluent ion should be considered as important factors in minimizing retention time shifts of the analyte. Thus, we can also define the proper time windows by a standard solution, which will undoubtedly shorten the time required for the determination of the time windows.

\section{References}

[1] R.M. Liu, B.T. Sun, I. Johns, Analyst 120 (1995) 2845.

[2] N. Revelante, M. Gilmartin, Mar. Biol. 34 (1976) 259.

[3] S.M. Hossein, R.M. Cassidy, J. Chromatogr. A 749 (1996) 279.

[4] L. Arce, A. Rios, M. Valcarcel, J. Chromatogr. A 791 (1997) 279.

[5] I. Ivancic, D. Degobbis, Wat. Res. 18 (1984) 1143.

[6] J. Kanda, Wat. Res. 29 (1995) 2746.

[7] A.J. Kempers, C.J. Kok, Anal. Chimi. Acta 221 (1989) 147

[8] D.P. Nikolelis, C.G. Siontorou, U.J. Krull, P.L. Katrivanos, Anal. Chem. 68 (1996) 1735.

[9] C. Jimenez, L. Marques, J. Bartroli, Anal. Chem. 68 (1996) 3801.

[10] D. Liu, R.K. Meruva, R.B. Brown, M.E. Meyerhoff, Anal. Chimi. Acta 321 (1996) 173.

[11] R.M. Liu, B.T. Sun, D.J. Liu, A.L. Sun, Talanta 43 (1996) 1049.

[12] L.S. Simeral, Appl. Spectr. 51 (1997) 1585.

[13] G. Zhang, P.K. Dasgupta, Anal. Chem. 61 (1989) 408

[14] R.M. Liu, H.S. Wang, A.L. Sun, D.J. Liu, Talanta 45 (1997) 405.

[15] H. Small, in: Ion Chromatography, Plenum Press, New York, 1989, pp. 219-221.

[16] M.A. Rey, J.M. Riviello, C.A. Pohl, J. Chromatogr. A 789 (1997) 149.

[17] M.A. Rey, C.A. Pohl, J.J. Jagodzinski, E.Q. Kaiser, J. Chromatogr. A 804 (1998) 201.

[18] J.K. Killgore, S.R. Villasenor, J. Chromatogr. A 739 (1996) 43.

[19] S. Peldszus, P.M. Huck, S.A. Andrews, J. Chromatogr. A 793 (1998) 198.

[20] S.R. Villasenor, J. Chromatogr. A 602 (1992) 155.

[21] P. Campins-Falco, R. Herraez-Hernandez, A. Sevillano-Cabeza, Anal. Chem. 66 (1991) 244.

[22] Y. Huang, S.F. Mou, Y. Yan, J. Liq. Chromatogr. Rel. Tech. in press.

[23] M. Novic, B. Divjak, B. Pihlar, V. Hudnik, J. Chromatogr. A 739 (1996) 35.

[24] H. Small, in: Ion Chromatography, Plenum Press, New York, 1989, Chapter 2.

[25] M. Maruo, N. Hirayama, T. Kuwamoto, J. Chromatogr. 481 (1989) 315. 
[26] H. Small, in: Ion Chromatography, Plenum Press, New York, 1989, p. 179 .

[27] H. Small, in: Ion Chromatography, Plenum Press, New York, 1989, Chapter 4.

[28] A.C.M. Brando, W.W. Buchberger, E.C.V. Butler, P.A. Fagan, P.R. Haddad, J. Chromatogr. A 706 (1995) 271.
[29] Marheni, P.R. Haddad, A.R. Mctaggart, J. Chromatogr. 546 (1991) 221.

[30] M. Rey, C. Pohl, J. Chromatogr. A 739 (1996) 87.

[31] D.P. Hautman, D.J. Munch, in: US EPA Method 300.1, 1997, pp. 14-15. 